\title{
Changes in head posture after rapid palatal expansion in mouth- breathing children.
}

\author{
Silvia Izabella Pop ${ }^{1}$, Martha Krisztina ${ }^{1}$, Laura Roxana Contac ${ }^{1}$, Sandor Henrietta ${ }^{1}$ \\ ${ }^{1}$ George Emil Palade University of Medicine, Pharmacy, Science, and Technology of Târgu-Mureș, Romania
}

\begin{abstract}
Introduction. It is well known that previously has been demonstrated a correlation between respiratory function and the harmonious craniofacial development and head posture respectively.

Aim of the study. The purpose of this study was to investigate the correlation between rapid maxillary expansion (RME) and the change in natural head position (NHP) resulting from the consequent change in airway resistance and to elucidate how RME influences NHP in terms of cephalometric angles in children with nasal obstruction.

Material and methods. The study included 12 girls and 8 boys who had a history of mouth breathing, as confirmed by the parents and ORL specialist and due to this fact, they underwent RME as part of orthodontic treatment. Dental casts, clinical photographs and lateral skull radiographs exposed in natural head position were obtained at the first visit (T1) and 8-10 months (T2) later for all subjects. In order to evaluate the patient's lateral skull radiographs, I used AudaxCeph software, within which I created a new type of analysis. Student T test and Pearson correlation test were used to statistically analyze the results.

Results. Our findings demonstrate that no significant changes in any of the variables are observed in patients treated with rapid maxillary expansion, however a positive correlation was observed in the variables measured before and after the treatment, respectively between the anomaly and the measured values. Positivity can also be detected when examining the correlation between the patient's gender and the measured values.

Conclusions. Based on the obtained results, we cannot state that there is a correlation between rapid maxillary expansion and cervical posture.

Keywords: head posture, children, nasal obstruction, rapid maxillary expansion, rapid palatal expansion.
\end{abstract}

\section{Introduction}

In the last few decades, the relationship between airway and the head posture was intensively examined by numerous researchers [1-3]. Clinicians are in complete agreement about the fact that, an important etiologic factor of the growth of the craniofacial structure is the respiratory function. In the orthodontic literature, Melvin Moss introduced, for the first time, the "functional matrix". He claimed that the facial development and growth of the individual is correlated to the functional activity of various structures of the head and neck, then it can be stated that oral respiration results in physiological changes of the facial skeleton and in addition in malocclusions [4-6].

In 1968 Ricketts has elucidated a connection between head posture and respiratory functional needs, demonstrating that head extension is a defensive compensatory functional mechanism that helps to keep the ability of the nasopharyngeal airways. Nasal obstruction is compensated by an altered position of tongue and soft palate which facilitates oral respiration [7].

Longitudinal studies, for example LinderAronson and co-workers have shown that patients with enlarged adenoids, tonsils, or other forms of airway obstruction have different craniofacial growth than patients who do not present airway obstruction in their history. Two months after adenoidectomy, Solow and Greve, as well as Woodside and Linder-Aronson, found a 2-degree decrease in craniocervical angle and the postural discrepancies between the control group and the children with various respiratory diseases were eliminated [8-11].The typical extraoral appearance of children with respiratory impairment caused by chronic nasopharyngeal obstruction is called "adenoid facies", which includes open mouth posture, hypotonic lips, compression of the upper arch, 
decreased transverse dimension of the maxilla, high palate vault, lateral occlusal bite and increased lower facial height [5]. Given that the typical malocclusion associated with respiratory obstruction is characterized by a transverse decrease in the palatal dimension, the primary method used to treat the insufficient transverse dimension of the maxillary base is rapid maxillary expansion (RME), which was introduced by Angell in the 1860s to treat maxillary compression [12-14]. RME is an effective procedure for correcting transverse discrepancies of the maxilla, exerting its enlargement effect on the jawbone through the "rupture/opening" of the medial palatine suture, resulting in the separation of the two maxilla halves to achieve true orthopedic expansion [15-17].

The use of RME was used by Derichsweiler and Korkhaus to increase airway patency. Gerlach advised that although RME is useful in nasal stenosis, not all mouth breathers can be treated this way [1, 18]. Linder-Aronson and Aschan, Hershey et al., Loreille and Béry showed evidence of an improvement in nasal respiration in patients treated with RME, revealing an average 13.6 percent increase in nasal permeability [19, 4]. In a study of 26 patients, Timms observed a 36.2 percent decrease in airway resistance after RME [20, 21].

The aim of the study was to evaluate the cephalometric points, planes and angles determining the head posture on the lateral radiographs of the selected patients with upper jaw compression, and to evaluate and compare the measured results of the initial state and the changes in the angular values after RPE.

\section{Materials and methods}

The sample

The observational, retrospective study included lateral skull radiographs of 20 patients with ages between 8-13 and a gender distribution of $60 \%$ female and $40 \%$ male. The radiographs of the patients were selected taking into consideration that the patients had no previous orthodontic treatment and the diagnostic included maxillary compression. The orthodontic treatment included rapid maxillary expansion, and a history of oral respiration was confirmed, in all of the cases. All of the patients parents signed an informed consent

The device was a Hyrax or a Haas type expander, activated 2 times a day by adjusting $1 / 4$ turn on the screw in the middle of the device with the special activation key in the direction of the arrow on the screw until the desired maxilla expansion was achieved. Activation of the device took an average of 10 14 days, after which the screw was blocked, and the device was left in place for 3 months to achieve the callus mineralization in the area of the median palatine suture.

\section{Clinical recordings}

The lateral head skull radiographs of all the 20 patients, were achieved at the first visit (T1) and the second record 8-10 months later. The heads of the patients were fixed in a cephalostat when the radiographs were taken. The mentioned digital X-rays were taken with a Pax Flex 3D +, Vatech X-ray machine with an exposure time of 12.9 seconds, $80 \mathrm{kVp}$ and $9.0 \mathrm{~mA}$. The dental imaging software used was EasyDent at DR X-RAY in Târgu Mureș. The orthodontic cephalometric program used in the study was AudaxCeph software.

A dossier was prepared for every patient and includes the personal data and the x-rays taken before and after the treatment was finalized. Since not all analysis types included in the software analysis type list contain all the elements needed to evaluate head posture, a new type of analysis was created for the study within the AudaxCeph software. The analysis included the angular and linear measurements suggested by Tecco et al.[4]. The X-ray images were calibrated using the cephalostat that appears on them. For the analysis of the head posture, the necessary cephalometric points based on the corresponding anatomical structure were first traced. As a second step, the planes and lines necessary for the analysis from the various combinations of the 
cephalometric points listed above were created and diverse variables resulting from the union of the cephalometric lines were measured (Figure 1).

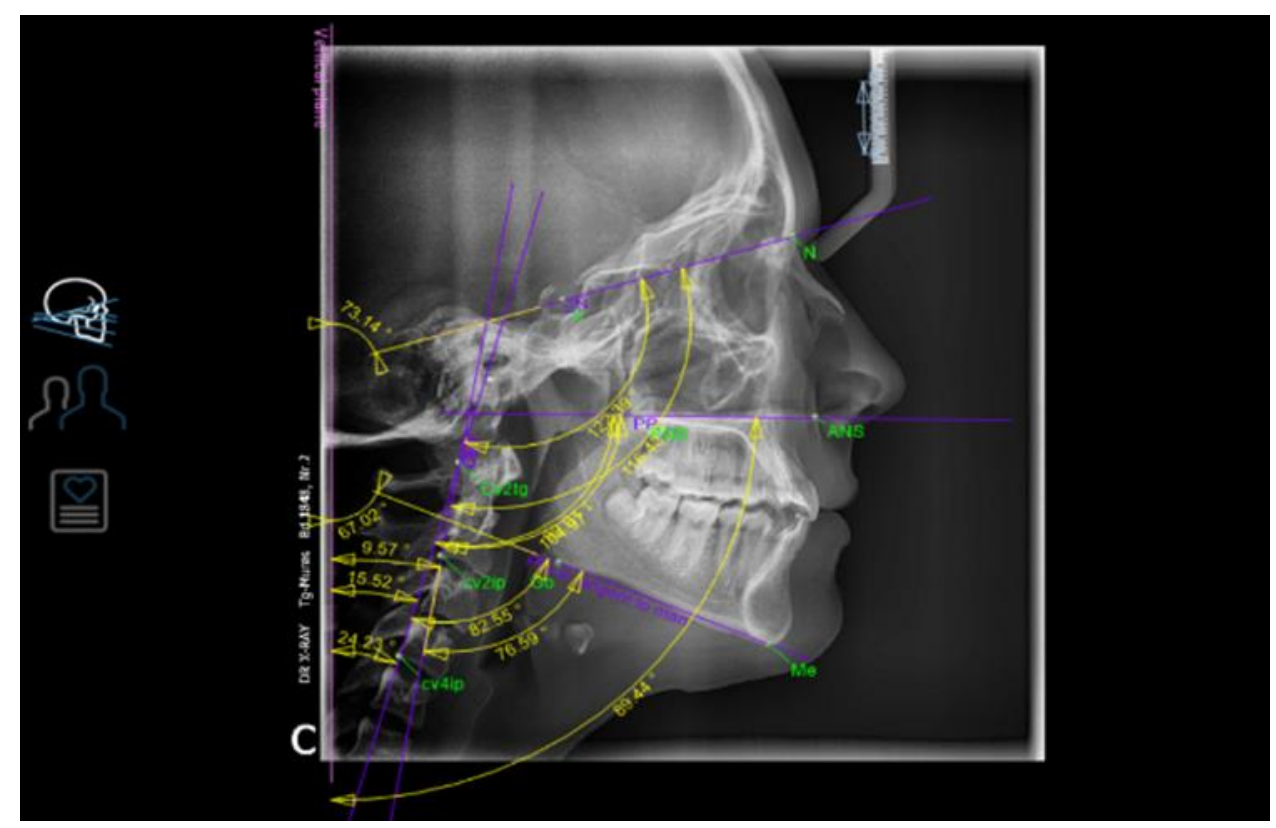

Figure 1. The cephalometric points traced on the lateral skull radiographs.

The points mentioned above: S (Sella Turcica), N (Nasion), PNS (Posterior Nasal Spine), ANS (Anterior nasal spine), Go (Gonion), Me (Menton), Cv2tg: the tangent point of the superior, posterior extremity of the odontoid process of the second cervical vertebra. Cv2ip: the most inferior and posterior point on the corpus of the second cervical vertebra. Cv4ip: the most infero-posterior point on the body of the fourth vertebra.

The cephalometric lines traced on the lateral skull radiographs were those defined and cited by Tecco et al."CVT: the upper part of the cervical spine. A line through cv2tg and cv4ip. OPT: odontoid line. A line through cv2tg and cv2ip. SN: anterior cranial base. A line through point $\mathrm{S}$ and point N. PP: palatal plane. A line through posterior nasal spine (PNS) and anterior nasal spine (ANS). MP: mandibular plane. A tangent line to the inferior border of the mandible. VER: true vertical plane.

OPT/VER: odontoid angle. The downward opening angle between the OPT and true vertical lines. EVT/VER: lower cervical column angle. The downward opening angle between the lower part of the cervical spine and true vertical lines. CVT/VER: upper cervical column angle. The downward opening angle between the CVT and true vertical lines. SN/VER: the downward opening angle between the $\mathrm{SN}$ and true vertical lines. PP/VER: the downward opening angle between the palatal lines and true vertical lines. MP/VER: the downward opening angle between the mandibular lines and true vertical lines. SN/OPT: the downward opening angle between OPT and SN lines. SN/CVT: the downward opening angle between CVT and $\mathrm{SN}$ lines. PP/OPT: the downward opening angle between OPT and palatal lines. PP/CVT: the downward opening angle between CVT and palatal lines. MP/OPT: the downward opening angle between OPT and mandibular lines. MP/CVT: the downward opening angle between CVT and mandibular lines" [4].

Completing these, the different variables were grouped, the first group being the cervical inclination, including OPT / VER and CVT / VER variables, while the second group contained the craniofacial inclination angles, these are SN / VER, PP / VER, and MP / VER. The craniocervical angles represent the 
third group constituted by the angles SN / OPT, SN / CVT, PP / OPT, PP / CVT, MP / OPT, and MP / CVT. Once the setup and analysis were performed, the software provided the results (the values obtained from that patients). All the cephalometric analysis were made by an experienced and trained orthodontist.

Using the saved measurements, the pre- and post-treatment angle values were analyzed for the patients both individually and collectively. Statistical analysis was performed using the Student $\mathrm{T}$ test and Pearson correlation test, at a level of significance $\mathrm{p} \leq 0.05$. Descriptive statistics was also performed using GraphPad Prism 5.0 for Windows.

\section{Results}

The 20 patients (12 females, 8 males) who enrolled in the study had a cephalometric radiograph taken before and after the treatment (8-10 months later). The measurements obtained in cephalometric analyses are presented in Tables 1-4.

Tabele 1. Changes with Treatment.

\begin{tabular}{|c|c|c|c|c|c|c|c|c|c|}
\hline & \multicolumn{2}{|c|}{ Pretreatment } & \multicolumn{2}{|c|}{ Posttreatment } & \multicolumn{5}{|c|}{ Change with Treatment } \\
\hline & Mean & SD & Mean & SD & Mean & SD & Minimum & Maximum & Significance \\
\hline \multicolumn{10}{|c|}{ Cervical inclination } \\
\hline OPT/Ver $\left({ }^{\circ}\right)$ & 7.24 & 5.07 & 7.46 & 5.89 & -0.22 & 5.71 & -4.3 & 3.86 & 0.91 \\
\hline CVT/Ver $\left({ }^{\circ}\right)$ & 7.73 & 5.37 & 9.57 & 6.06 & -1.83 & 5.47 & -6.04 & 2.37 & 0.34 \\
\hline \multicolumn{10}{|c|}{ Craniofacial inclination } \\
\hline SN/Ver $\left({ }^{\circ}\right)$ & 97.64 & 8.63 & 96.64 & 9.16 & 1 & 5.17 & -2.7 & 4.7 & 0.56 \\
\hline PP/Ver $\left({ }^{\circ}\right)$ & 93.29 & 5.77 & 92.13 & 2.87 & 1.16 & 5.3 & -2.63 & 4.95 & 0.51 \\
\hline MP/Ver $\left({ }^{\circ}\right)$ & 72.11 & 17.85 & 66.33 & 4.7 & 5.78 & 17.76 & -6.92 & 18.48 & 0.33 \\
\hline \multicolumn{10}{|c|}{ Craniocervical inclination } \\
\hline SN/OPT $\left({ }^{\circ}\right)$ & 100.83 & 11.69 & 101.67 & 12.37 & -0.84 & 6.37 & -5.4 & 3.72 & 0.69 \\
\hline $\mathrm{SN} / \mathrm{CVT}\left({ }^{\circ}\right)$ & 104.64 & 11.13 & 106.53 & 12.86 & -1.89 & 5.67 & -6.24 & 2.47 & 0.35 \\
\hline $\mathrm{PP} / \mathrm{OPT}\left({ }^{\circ}\right)$ & 93.08 & 10.52 & 93.78 & 9.68 & -0.7 & 6.26 & -5.18 & 3.78 & 0.73 \\
\hline $\mathrm{PP} / \mathrm{CVT}\left({ }^{\circ}\right)$ & 96.84 & 9.88 & 98.36 & 9.72 & -1.51 & 5.39 & -5.66 & 2.63 & 0.42 \\
\hline $\mathrm{MP} / \mathrm{OPT}\left({ }^{\circ}\right)$ & 67.11 & 9.34 & 67.99 & 9.01 & -0.88 & 7.47 & -6.22 & 4.46 & 0.72 \\
\hline $\mathrm{MP} / \mathrm{CVT}\left({ }^{\circ}\right)$ & 70.33 & 9.17 & 71.97 & 8.74 & -1.63 & 6.46 & -6.6 & 3.33 & 0.47 \\
\hline
\end{tabular}

Table 1 shows the mean and standard deviation of each variable measured at the beginning (Pretreatment) and end of treatment (Posttreatment). It also describes the changes which occurred with the treatment.

The cervical inclination angles, OPT/Ver and CVT/ Ver, both increased mildly when measured at the end of the treatment, but this change was not statistically significant. Craniofacial inclination angles, represented by $\mathrm{SN} /$ Ver, PP/Ver, MP/Ver, showed a slightly decreasing between pre- and posttreatment, but neither of the changes were found to be statistically relevant. A slight increase can also be observed in the angles of craniocervical inclination (SN/OPT, SN/CVT, PP/OPT, $\mathrm{PP} / \mathrm{CVT}, \mathrm{MP} / \mathrm{OPT}, \mathrm{MP} / \mathrm{CVT})$, however these angulations were not found to be significant statistically. The results of our study shows that for the patients treated with RME there is no significant change in any of the variables, however in the study of the longterm effects of RPE on nasopharyngeal airway size, head posture, and cervical curvature angle of Tecco et al from pre- to posttreatment, the study group had a significant $(\mathrm{p}<.001) 3.67^{\circ}$ backward inclination of the upper cervical column (OPT/ Ver angle). Twenty-three female patients were included in the study group. For the girls with nasal obstruction under active treatment there was a statistically significant flexion of the head, this is evidenced by the decreased craniofacial inclination angles $\left(5.25^{\circ}, \mathrm{P}<.0001\right.$ for $\mathrm{SN} /$ Ver angel; 5.04 , 
$\mathrm{P}<.0001$ for $\mathrm{PP} /$ Ver angle; $4.40^{\circ}, \mathrm{P}<.0001$ for $\mathrm{MP} /$ Ver). A significant decreased of $5.1^{\circ}$, $4.36^{\circ}$, and $5.12^{\circ}$ in the mean craniocervical angles (SN/OPT, PP/OPT, and MP/OPT) was also observed. Our research has succeeded to shown that a positive correlation exists in the variables measured before and after the treatment (Table 2).

Table 2. Correlations between the variables measured before and after the treatment.

\begin{tabular}{|c|c|c|}
\hline & Correlation & Significance \\
\hline \multicolumn{3}{|l|}{ Cervical inclination } \\
\hline OPT/Ver $\left({ }^{\circ}\right)$ & 0.466 & 0.174 \\
\hline CVT/Ver $\left({ }^{\circ}\right)$ & 0.547 & 0.127 \\
\hline \multicolumn{3}{|c|}{ Craniofacial inclination } \\
\hline SN/Ver $\left({ }^{\circ}\right)$ & 0.833 & 0.003 \\
\hline $\operatorname{PP} / \operatorname{Ver}\left({ }^{\circ}\right)$ & 0.407 & 0.244 \\
\hline $\mathrm{MP} / \operatorname{Ver}\left({ }^{\circ}\right)$ & 0.15 & 0.679 \\
\hline \multicolumn{3}{|c|}{ Craniocervical inclination } \\
\hline SN/OPT $\left({ }^{\circ}\right)$ & 0.861 & 0.001 \\
\hline $\mathrm{SN} / \mathrm{CVT}\left({ }^{\circ}\right)$ & 0.898 & 0.001 \\
\hline $\mathrm{PP} / \mathrm{OPT}\left({ }^{\circ}\right)$ & 0.811 & 0.004 \\
\hline $\mathrm{PP} / \mathrm{CVT}\left({ }^{\circ}\right)$ & 0.849 & 0.004 \\
\hline $\mathrm{MP} / \mathrm{OPT}\left({ }^{\circ}\right)$ & 0.669 & 0.034 \\
\hline $\mathrm{MP} / \mathrm{CVT}\left({ }^{\circ}\right)$ & 0.741 & 0.022 \\
\hline
\end{tabular}

Of the craniofacial inclination angles, the $\mathrm{SN} / \operatorname{Ver}(\mathrm{r}=0.833$, at $\mathrm{P}<0.003)$ angle is the one at which the positive correlation can be detected, while all the craniocervical inclinations angles show a significant correlation, SN/OPT $(\mathrm{r}=0.861, \quad \mathrm{P}<0.001)$, $\mathrm{SN} / \mathrm{CVT} \quad(\mathrm{r}=0.898, \quad \mathrm{P}<0.001), \quad \mathrm{PP} / \mathrm{OPT}$ $(\mathrm{r}=0.811, \quad \mathrm{P}<0.004), \quad \mathrm{PP} / \mathrm{CVT} \quad(\mathrm{r}=0.849$, $\mathrm{P}=0.004), \mathrm{MP} / \mathrm{OPT}(\mathrm{r}=0.669, \mathrm{P}<0.034)$, and $\mathrm{MP} / \mathrm{CVT}(\mathrm{r}=0.741, \mathrm{P}<0.022)$. Positivity can also be detected when examining the correlation between the anomaly and the measured values (Table 3).

Table 3. Correlations between the anomaly and the measured values.

\begin{tabular}{|c|c|c|}
\hline & Correlation & Significance \\
\hline \multicolumn{3}{|l|}{ Cervical inclination } \\
\hline OPT/Ver $\left({ }^{\circ}\right)$ & 0.439 & 0.277 \\
\hline CVT/Ner $\left({ }^{\circ}\right)$ & 0.56 & 0.149 \\
\hline \multicolumn{3}{|l|}{ Craniofacial inclination } \\
\hline SN/Ver $\left({ }^{\circ}\right)$ & 0.723 & 0.043 \\
\hline $\operatorname{PP} / \operatorname{Ver}\left({ }^{\circ}\right)$ & 0.396 & 0.331 \\
\hline $\operatorname{MP} / \operatorname{Ver}\left({ }^{\circ}\right)$ & 0.099 & 0.815 \\
\hline \multicolumn{3}{|l|}{ Craniocervical inclination } \\
\hline SN/OPT $\left({ }^{\circ}\right)$ & 0.896 & 0.003 \\
\hline $\mathrm{SN} / \mathrm{CVT}\left({ }^{\circ}\right)$ & 0.914 & 0.914 \\
\hline $\mathrm{PP} / \mathrm{OPT}\left({ }^{\circ}\right)$ & 0.873 & 0.005 \\
\hline $\mathrm{PP} / \mathrm{CVT}\left({ }^{\circ}\right)$ & 0.867 & 0.005 \\
\hline $\mathrm{MP} / \mathrm{OPT}\left({ }^{\circ}\right)$ & 0.824 & 0.012 \\
\hline $\mathrm{MP} / \mathrm{CVT}\left({ }^{\circ}\right)$ & 0.83 & 0.011 \\
\hline
\end{tabular}


The downward opening angle between the $\mathrm{SN}$ and true vertical lines is correlated with nasal obstruction $(\mathrm{r}=0.723, \mathrm{p}<0.043)$. Among the angles of craniocervical inclination that show a positive correlation with the anomaly, we can mention the SN/OPT $(r=0.896$, $\mathrm{P}<0.003), \quad \mathrm{PP} / \mathrm{OPT} \quad(\mathrm{r}=0.873, \quad \mathrm{P}<0.005)$, $\mathrm{PP} / \mathrm{CVT} \quad(\mathrm{r}=0.867, \quad \mathrm{P}<0.005), \quad \mathrm{MP} / \mathrm{OPT}$ $(\mathrm{r}=0.824, \quad \mathrm{P}<0.012), \quad \mathrm{MP} / \mathrm{CVT} \quad(\mathrm{r}=0.83$, $\mathrm{P}<0.011)$.

There is a positive correlation between the patient's gender and the measured values (Table 4), which is evidenced by the SN/ Ver $(\mathrm{r}=0.853, \quad \mathrm{P}<0.007)$ from the craniofacial inclination angles shown in Table 4, respectively SN/OPT $(\mathrm{r}=0.878, \quad \mathrm{P}<0.004)$, $\mathrm{SN} / \mathrm{CVT} \quad(\mathrm{r}=0.905, \quad \mathrm{P}<0.002), \quad \mathrm{PP} / \mathrm{OPT}$ $(\mathrm{r}=0.852, \quad \mathrm{P}<0.007), \quad \mathrm{PP} / \mathrm{CVT} \quad(\mathrm{r}=0.858$, $\mathrm{P}<0.006), \mathrm{MP} / \mathrm{OPT}(\mathrm{r}=0.726, \mathrm{P}<0.041)$, and $\mathrm{MP} / \mathrm{CVT}(\mathrm{r}=0.751, \mathrm{P}<0.032)$ angles, which represent the group of craniocervical inclination.

\section{Discussion}

One of the most researched topics of the current orthodontic literature is the treatment results of the rapid maxillary expansion on the dentofacial morphology. The increase in palatal width may result in enlargement of the pharyngeal airway space, improved respiratory function, and changes of the head posture on the cervical column, with an increase in the cervical curvature angle and a decrease in craniocervical angulation, according to a potential hypothesis for the role of RME in postural alterations [1, 22, 23].

Following different forms of treatments that enhance nasal respiratory function, several researchers have documented substantial improvements in head position and craniocervical angulation. In a recent case report the authors theorized that the changes in palatal width acquired with RME appliances might influence additional bone structures such as the tongue's muscles and the suboccipital muscles [24, 25].
The objective of this study was to evaluate the effects of rapid maxillary expansion on head posture. Changes in pretreatment and posttreatment were measured using lateral cephalometric radiographs. Both pre- and posttreatment radiographs were taken in natural head position, with the appliance removed.

The result of our study shows no significant changes after the RME in angles which are used to determine head posture. Our findings are not in accordance with those of Tecco et al, who studied the changes of cervical posture following palatal expansion in 45 mouth breathing girls. In Tecco study patients from 8 to 15 years of age were included: 23 subjects in the study group and 22 children in the control group. Lateral cephalograms were obtained at the first visit, respectively 6 and 12 months later for all subjects. 6 months after expansion of the palate, there was a significant backward inclination of the cervical column in the study group (mean increase of $3.67^{\circ} ; \mathrm{P}<0.05$ ). The changes observed in flexion of the head after one year in the test group were also significant $\left(5.25^{\circ}\right.$ for $\mathrm{SN} /$ Ver angle, $5.04^{\circ}$ for $\mathrm{PP} /$ Ver angle, $4.40^{\circ}$ for $\mathrm{MP} / \mathrm{Ver}$; all $\mathrm{P}<0.05$ ). Craniocervical angles, measured by SN/OPT, $\mathrm{PP} / \mathrm{OPT}$, and MP/OPT, decreased by 5.1, 4.36 and 5.12 degrees in the study group after 6 months $(\mathrm{P}<0.05)$ [4].

Kjurchieva-Chuchkova et al. observed a statistically significant change in the head posture and decrease in craniocervical angulation, especially at the downward opening angle between OPT and palatal lines (4.07, for PP-OPT angle) and angle interaction between the upper part of the cervical spine and palatal plane (4.95 degrees for PP/CVT angle). The findings of this study are not in accordance with our investigation [5].

Our results are corresponding with those of longitudinal studies of Yagci et al, who have investigated the effects of rapid palatal expansion on natural head position in 38 subjects. The treatment group comprised 23 patients and the control group 15 patients. The 
NHP data was collected using an inclinometer and a portable data logger. Both the study and control group had NHP recordings taken at the beginning of appliance placement and at the completion of RME therapy. Similar to our results, was no statistically significant difference between initial and final NHP, the mean difference pre-and posttreatment for NHP was $0.31^{\circ}$ [26].

The findings of our study confirmed a positive correlation between the measured variables and the gender of the patient, evidenced by the angles SN/Ver, SN/OPT, $\mathrm{SN} / \mathrm{CVT}$, PP/OPT, PP/CVT, MP/OPT, $\mathrm{MP} / \mathrm{CVT}$, respectively between the anomaly and the measurements, proved by the $\mathrm{SN} / \mathrm{Ver}$, SN/OPT, PP/OPT, PP/CVT, MP/OPT, and $\mathrm{MP} / \mathrm{CVT}$ variables.

Although there is a modification in head position after RME, it cannot be assessed objectively since the few studies that have been published do not adopt the same evaluation approach. One of the shortcomings of our study is the reduced number of patients. To provide a more accurate result, more wellcontrolled long-term clinical trials utilizing the most accurate methods to determine craniocervical morphology and function, as well as a longer observation time, are needed.

\section{Conclusion}

Based on the obtained results, we cannot state that there is a correlation between rapid maxillary expansion and cervical posture.

Conflict of interest: None to declare.

\section{References}

1. Niall JPM, McGuinness J. Changes in natural head position observed immediately and one year after rapid maxillary expansion. Eur. J. Orthod. 2006;28(2):126-34

2. Sonnensen L, Peterson A, Berg S. Pharyngeal Airway Dimensions and Head Posture in Obstructive Sleep Apnea Patients with and without Morphological Deviations in the Upper Cervical Spine. J. Oral Maxillofac. Surg. 2017;8(3): e4
3. Al Taki A, Thabit A. Changes in Pharyngeal Airway Dimensions, Hyoid position, and Head Posture after Rapid Palatal Expansion and Face Mask Therapy. Am. J. Sci. 2014;10(10):259-26

4. Tecco S, Caputti S, Festa F. Evaluation of cervical posture following palatal expansion: a 12-month follow-up controlled study. Eur. J. Orthod. 2007;29(1):45-51

5. Kjurchieva-Chuchkova G, Kanurkova L, Bajraktarova MC. Changes in head posture after rapid maxillar expansion in patients with nasopharyngeal obstruction. South Eur J Orthod Dentofac Res. 2016;3(2):39-43

6. Kyrkanides S, Moore T, Miller JH, Tallents RH. Melvin Moss' function matrix theory-Revisited. Orthod Waves. 2011;70(1):1-7

7. Cuccia AM, Lotti M, Carradonna D. Oral Breathing and Head Posture. Angle Orthod. 2008;78(1):7782

8. Mehta S, Lodha S, Valiathan A, Urala A. Mandibular morphology and pharyngeal airway space: A cephalometric study. APOS Trends Orthod. 2014;5(1):22-28

9. Valcheva Z, Aranutska $H$, et al. Epidemiological research on the incidence of malocclusions among mouth-breathing children with primary and mixed dentition. J Union Sci - Varna. 2018;23:127-133

10. Arun T, Isik F, Sayinsu K. Vertical Growth Changes After Adenoidectomy. Angle Orthod. 2003;73(2):146-50

11. Solow B, Sandham A. Cranio-cervical posture: a factor in the development and function of the dentofacial structures. Eur. J. Orthod. 2002;24:447-456

12. Agarwal A, Mathur R. Maxillary Expansion. Int. J. Paediatr. Dent. 2010;3(3):139-46

13. Zuccati G, Casci S, Doldo T, Clauser C. Expansion of maxillary arches with crossbite: a systematic review of RCTs in the last 12 years. Eur. J. Orthod. 2013;35(1):29-37

14. Baccetti T, Franchi L, Cameron CG, McNamara JA Jr. Treatment timing for rapid maxillary expansion. Angle Orthod. 2001;71(5):343-350

15. Mundstock KS, Barreto G, Meloti AF, Araújo MA, dos Santos-Pinto A, Raveli DB. Rapid maxillary expansion with the Hyrax appliance: an occlusal radiographic evaluation study. World J Orthod.2007;8(3):277-84

16. Franchi L, Baccetti T, Lione R, Fanucci E, Cozza P. Modifications of midpalatal sutural density induced by rapid maxillary expansion: A low-dose computed-tomography evaluation. Am J Orthod Dentofacial Ortho. 2010;137(4):486-8 
17. Tecco S, Festa F, Tete S, Longhi V, D'Attilioet M. Changes in Head Posture after Rapid Maxillary Expansion in Mouth-Breathing Girls: A Controlled Study. Angle Orthod. 2005;75(2):171-6

18. Provatidis CG, Georgiopoulos B, Kotinas A. Evaluation of craniofacial effects during rapid maxillary expansion through combined in vivo/in vitro and finite element studies. Eur. J. Orthod. 2008;30:437-480

19. Chiari S, Romsdorfer P, Swoboda H, Bantleon HP, Freudenthaler J. Effects of rapid maxillary expansion on the airways and ears - a pilot study. Eur. J. Orthod. 2009;31 (2):135-141

20. Kiliç $N$, Oktay $H$. Effects of rapid maxillary expansion on nasal breathing and some nasorespiratory and breathing problems in growing children: A literature review. Int. J. Pediatr. Otorhinolaryngol. 2008;72(11): 1595-1601

21. Aloufi F, Preston CB, Zawawi KH. Changes in the upper and lower pharyngeal airway spaces associated with rapid maxillary expansion. ISRN Dentistry. 2012. doi: 10.5402/2012/290964
22. Matsumoto NAM, Itikawa CE, Pereira FCV, Faria G, Anselmo-Lima WT. Long-Term Effects of Rapid Maxillary Expansion on Nasal Area and Nasal Airway Resistance. Am J Rhinol Allergy. 2010; 24(2):161-5

23. Yagci A, Uysal T, Usumez S, Orhanet M. Rapid maxillary expansion effects on dynamic measurement of natural head position. Angle Orthod. 2011; 81(5):850-855

24. Monaco A, Tepedino M, Sabetti L, Petrucci A, Sgolastra F. An adolescent treated with rapid maxillary expansion presenting with strabismus: a case report. J Med Case Rep. 2013;7(1):222

25. De Felippe NLO, Da Silveira AC, Viana G, Kusnoto B, Smith B, Evans AC. Relationship between rapid maxillary expansion and nasal cavity size and airway resistance: short- and long-term effects. Am J Orthod Dentofac Orthop. 2008;134(3):37082

26. Yagci A, Uysal T. Effects of conventional and modified facemask therapies on dentofacial structures. Korean J Orthod. 2010;40(6):432-443

\section{Corresponding author:}

Laura Roxana Contac

George Emil Palade University of Medicine, Pharmacy, Science and Technology of Târgu -Mureș, 38 Gheorghe Marinescu Street, Târgu -Mureș, 540139, Romania

Email: lauracontac92@gmail.com 